Strom an der Kathode eines Hochdruckbogens von Ionen gedeckt wird, durchgerechnet und nehmen dicht vor der Kathode ein thermisch ionisiertes Gebiet hoher Temperatur an. Die von Haynes ${ }^{3}$ beobachtete Richtwirkung im Dampfstrahl ist jedoch durch diese Theorie nicht zu verstehen. Interessant ist die Tatsache, daß van Voorhis und Compton $^{8-10}$ für $\mathrm{He}, \mathrm{Ne}$ und $\mathrm{A}$ und Larché ${ }^{11}$ für einen Edelgashochdruckbogen einen von den nicht verdampfenden Kathoden ausgehenden Gasstrahl finden. Demnach spricht vieles dafür, daß das Gebiet

${ }^{8}$ Vanyoorhis u. K. J. Compton, Physic. Rev. 37, 1596 [1931].

9 E. S. Lamar, Physic. Rev. 37, 842 [1931]; Physic. Rev. 43, 169 [1933].

10 K. J. Compton u. E. S. Lamar, Physic. Rev. 44, 338 [1933].

11 K. Larché, Lichttechn. 2, 41 [1950].

${ }^{12}$ L. B. Loeb, Fundamental Processes of Electrical Discharge in Gases, J. Wiley \& Sons, Inc., London, New York [1939]. der Strahlerzeugung im Kathodenfall selbst zu suchen ist und daß es sich bei diesen schnellen Dampfteilchen im Sinne von Compton ${ }^{10}$, Loeb $^{12}$ und Haynes ${ }^{3}$ um ursprünglich auf die Kathode zufliegende Ionen handelt, die bei ihrer Neutralisation an der Kathode teilweise reflektiert werden. Zumindest größenordnungsmäßig läßt sich die von uns gemessene Geschwindigkeit auf diese Weise erklären; denn bei einem Kathodenfall von rund $6 \mathrm{~V}$ liegt die Auftreffgeschwindigkeit von Hg-Ionen bei etwa $2,5 \cdot 10^{5} \mathrm{~cm} / \mathrm{sec}^{13}$.

13 Herr J. v. Isse nd orff weist uns freundlicherweise darauf hin, daß der von uns untersuchte Dampfstrahl möglicherweise nicht im Brennfleck selbst, sondern ähnlich wie bei seinen Messungen der verdampften Hg-Menge (Physik. Z. 19, 857 [1928]) von der durch den Erregerbogen aufgeheizten $\mathrm{Hg}$-Oberfläche ausgeht. Dagegen sprechen jedoch die thermisch unverständlich hohen Geschwindigkeiten und besonders die von Haynes ${ }^{3}$ nachgewiesene ausgesprochene Richtwirkung.

\title{
Über die Entmischung von Lösungen des Polymethacrylesters II $^{1}$
}

\author{
Von E. Jenckel und J. Delahaye \\ Aus dem Institut für theoretische Hüttenkunde und physikalische Chemie \\ der Techn. Hochschule Aachen \\ (Z. Naturforschg. 7a, 682-692 [1952]; eingegangen am 19. April 1952)
}

\begin{abstract}
Es wurden die Löslichkeitskurven einer Anzahl von Fraktionen des Polymethacrylsäuremethylesters mit Polymerisationsgraden von 500 bis 30000 in $p$-Cymol, $n$-Propanol und $n$-Butanol bestimmt.

Es wurden die Koordinaten des kritischen Lösůngspunktes thermodynamisch berechnet. Hierbei bewährt sich vorzüglich ein Ansatz für die Mischungsentropie athermischer Lösungen von Miller in Verbindung mit einem quadratischen Ausdruck $\mu x_{2}^{2}$ für die Lösungswärme und die zusätzliche Entropie der irregulären Lösung, in dem $\mu=\alpha+\beta / T$ zu setzen ist.
\end{abstract}

$\mathrm{F}$ ür binäre Lösungen aus hochmolekularen und niedrigmolekularen Stoffen, die sich beim Abkühlen in zwei flüssige Phasen entmischen, ist es kennzeichnend, daß die kritische Zusammensetzung sehr weit zur Seite des reinen Lösungsmittels verschoben ist. Die vorliegende Arbeit untersucht im Anschluß an eine frühere ${ }^{1}$ Mitteilung solche Löslichkeitskurven an Polymethacrylester. In der früheren Arbeit wurde versucht, die Koordinaten des kritischen Lösungspunktes thermodynamisch zu berechnen. Diese thermodynamische Berechnung ist ein vorzügliches Kriterium dafür, ob die verwendeten Entropie- und Enthalpieformeln richtig sind

${ }^{1}$ Vgl. I: E. Jenckel u. K. Gorke, Z. Naturforschg. 5a, $556[1950]$. oder nicht. Die damals verwendeten Formeln nämlich, eine statistisch abgeleitete Entropieformel von Miller, Huggins und Flory in Verbindung mit einem quadratischen Ausdruck für die Lösungswärme, gab zwar viele Beobachtungen vorzüglich wieder, nicht aber die Abhängigkeit der kritischen Temperatur vom Polymerisationsgrad. Es wurde vermutet, daß die früher verwandten Fraktionen noch nicht genügend einheitlich seien und hierauf die Diskrepanz zwischen Theorie und Experiment zurückzuführen sei. In der vorliegenden Arbeit wurde besonders großer Wert auf sorgfältige und mehrfache Fraktionierung gelegt. Die beobachteten Löslichkeitskurven mit diesen Präparaten zeigen jedoch, daß der Einfluß einer geringeren oder größe- 
ren Einheitlichkeit die bisherige Diskrepanz nicht wesentlich beseitigt. Dagegen erzielt man eine vorzügliche Übereinstimmung, wenn man den beiden oben erwähnten Ansätzen ein bereits früher von Huggins und Flory verwendetes Zusatzglied anfügt. Aus den so entstehenden komplizierten Ausdrücken lassen sich gute Näherungsformeln entwickeln, die es erlauben, die kritischen Daten rasch anzugeben:

\section{A. Darstellung, Fraktionierung und Molekulargewicht des \\ Polymethacrylsäuremethylesters}

\section{Herstellung des Polymerisates}

Monomerer Ester* wurde bei $100 \mathrm{~mm} \mathrm{Hg}$ und $49^{\circ} \mathrm{C}$ destilliert. Seine Dichte betrug bei $18^{\circ} \mathrm{C} 0,940 \pm 0,02$ in hinreichender Übereinstimmung mit Literaturangaben. Der Ester wurde in Abschmelzrohren von etwa $60 \mathrm{~cm}$ Länge im Block polymerisiert und zwar auf zwei verschiedene Weisen. Ansatz I entstand bei $65^{\circ}$ bis $70^{\circ}$ innerhalb von 6 Tagen ohne Zusatz eines Katalysators. Das Polymerisat lag nach dem Zerschlagen der Abschmelzrohre als klar durchsichtige, aber sehr harte Stangen vor, die nur mit großer Mühe zerkleinert werden konnten. Im Ansatz II wurde bei $50^{\circ} \mathrm{C}$ und unter $\mathrm{Zu}$ satz von $1 / 2 \%$ Benzoylperoxyd als Katalysator über 22 Stdn. polymerisiert. Das Polymerisat fiel in Form von nicht sehr harten biegsamen Stangen an.

\section{Fraktionierung}

Es wurde versucht, den Ansatz I in Methylacetat zu lösen. Das Polymerisat quoll stark auf, löste sich aber nur sehr langsam. Schließlich gelang es nach 8 Tagen, die Konzentration auf etwa $8 \mathrm{~g}$ pro $l$ zu steigern. Daraufhin wurde der Lösungsversuch abgebrochen und diese Lösung zur Fraktionierung verwandt. Ansatz II wurde ohne Schwierigkeiten in Aceton gelöst.

Fraktioniert wurde durchweg durch Zusatz einer Mischung von Methanol mit 25\% Wasser. Dabei haben wir in jedem Falle den zuerst erhaltenen Niederschlag durch Erwärmen der Lösung wieder vollständig gelöst und ihn beim Abkühlen unter Rühren wieder ausfallen lassen. Es hat das den Vorteil einer viel gleichmäßigeren Ausfällung, während bei unmittelbarem Zusatz des Fällungsmittels stets örtlich Überkonzentrationen auftreten müssen. Im übrigen haben wir uns an die Arbeiten von G.V. Schulz ${ }^{2}$ gehalten und besonders darauf Wert gelegt, daß die Konzentration des Polymerisates in den Lösungen möglichst klein bleibt. Im einzelnen gingen wir folgendermaßen vor: Die Lösung in Methylacetat (Ansatz I) bzw. in Aceton (Ansatz II) wurde vollständig mit wässerigem Methanol ausgefällt, wobei nur das Monomere und die ganz kurzen Ketten in der Lösung zurückbleiben. Der Niederschlag wurde in Me-

* Der Badischen Anilin- und Sodafabrik danken wir für die Überlassung. thylacetat gelöst und mit wässerigem Methanol in die Fraktionen I A und II B bzw. II A, II B und II C zerlegt. Die weitere Fraktionierung von I A und I B machte Schwierigkeiten, indem bei einer gewissen Fällungsmittelkonzentration das gesamte Polymerisat wieder ausfiel, also eine weitere Fraktionierung nicht mehr erreicht wurde. Es gelang schließlich, aus einęm Teil von I A durch stärkére Verdünnung der Lösung eine Unterfraktion [1] abzutrennen. Der Rest der Fraktion I A wurde völlig ausgefällt, durch Erhitzen auf $40^{\circ} \mathrm{C}$ wieder gelöst und durch Abkühlen auf etwa $22^{\circ} \mathrm{C}$ eine weitere Unterfraktion [2] gewonnen. Aus deren Dekantat wurde schließlich durch Abkühlen bei Zimmertemperatur noch eine dritteUnterfraktion [2a] erhalten. Wir halten die zweite Fraktion für am einheitlichsten, indem wir annehmen, da $B$ alle langen Ketten sich in der Fraktion [1] und alle kürzeren Ketten in der Fraktion [2 a] angesammelt haben. Die Fraktion I B wurde nicht unterfraktioniert. Die Fraktionen II A, II B und II C wurden durch Ausfällen mit Fällungsmittel, Lösen durch Erwärmen und Entmischung infolge Abkühlung in je 2 Fraktionen zerlegt. Alle diese Unterfraktionen wurden noch einmal gelöst, gefällt und durch Erwärmen gelöst, aber nur bis auf etwa $22^{\circ} \mathrm{C}$ abgekühlt und auf diese Weise von den kurzen Anteilen befreit. Die allerletzte Fraktion dieses Ansatzes war durch Abdampfen der Lösungsmittel gewonnen worden. Sie wird vermutlich nicht besonders einheitlich sein.

Die so erhaltenen Fraktionen stellen nach dem Trocknen zähe, dichte und harte Massen dar, die nicht pulverisierbar sind und vermutlich auch noch Lösungs- und Fällungsmittel enthalten. Sie müßten durch Umfällen in eine lockere, für die Trocknung geeignete Form überführt werden. Hierzu wurden sie zu etwa $1 \%$ in Metylacetat gelöst; die Löșung ließ man unter Rühren in die etwa 3-fache Menge eines MethanolWassergemisches eintropfen. Um während des Eintropfens die Konzentrationsverhältnisse im wässerigen Methanol nicht zu ändern, läßt man zweckmäßigerweise gleichzeitig mit der Polymerisatlösung die 3fache Menge Methanol zutropfen. Nach dem Abdekantieren wird der Niederschlag mit Methanol ausgekocht, anschließend in Äther gewaschen und abgenutscht.Das Produkt ist im Mörser leicht zu feinem Pulver zu verreiben, das im Vakuum von $20 \mathrm{~mm} \mathrm{Hg}$ bei $60^{\circ} \mathrm{C}$ über Chlorcalcium getrocknet und im Exsikkator über Calciumchlorid aufbewahrt wurde.

So hergestellte Proben zeigten beim Erhitzen auf $190^{\circ} \mathrm{C}$ während 30 Min., wobei sie sich nach Grassie ${ }^{3}$ nicht zersetzen, einen Trocknungsverlust von stets weniger als $0,5 \%$.

\section{Bestimmung des Polymerisationsgrades}

Die Polymerisationsgrade wurden viskosimetrisch in einem Ostwald-Viskosimeter bestimmt. Die Kapillare war so gewählt, daß die Ausflußzeit des reinen Lösungsmittels Chloroform zwischen 60 und 120 Sek.

${ }^{2}$ G. V. Schulz, Z. physik. Chem., Abt. B 46, 137 [1940].

${ }^{3}$ N. Grassie, J. Polymer Sci. 6, 643 [1951]. 
lag. Die Konzentration wurde so genommen, daß die spezifische Viskosität etwa zwischen 0,2 und 1,6 betrug. Die Temperatur war mit einem Thermostaten auf $0,1^{0}$ konstant gehalten. Vor der Messung wurde die Lösung noch einmal filtriert, um sie staubfrei zu machen.

Aus der spezifischen Viskosität der Lösung

$$
\eta_{\mathrm{sp}}=\frac{\eta_{\mathrm{L}}-\eta_{0}}{\eta_{0}}
$$

$\left(\eta_{\mathrm{L}}\right.$ und $\eta_{0}=$ Viskosität der Lösung bzw. des Lösungsmittels, $t_{\mathrm{L}}$ und $t_{0}=$ Durchlaufzeit der Lösung bzw. des Lösungsmittels) muß man den Grenzwert für unendliche Verdünnung bestimmen. Das geschah nach Schulz und Blaschke ${ }^{4}$ nach der Formel

$$
Z_{\eta}=\lim _{c \rightarrow 0} \frac{\eta_{\mathrm{sp}}}{c}=\frac{\eta_{\mathrm{sp}}}{1+K_{\eta} \eta_{\mathrm{sp}}} \frac{1}{c},
$$

worin für $K_{\eta}$ der Wert 0,29 nach $\mathrm{Schulz}^{5}$ eingesetzt ist. Aus (2) läßt sich der Polymerisationsgrad angeben, und zwar nach Staudinger

$$
Z_{\eta}==K_{\mathrm{m}} P
$$

und nach $\mathrm{Kuhn}$

$$
Z_{\eta}=K P^{n}
$$

Wir bevorzugen die Darstellung nach Kuhn und setzen mit $\mathrm{Schulz}^{6}$

$$
Z_{\eta}=5,1 \cdot 10^{-6} \cdot M^{0,81} .
$$

Da das Molekulargewicht des Grundmoleküls 100 ausmacht, ist der Polymerisationsgrad $P$ 1/100 des Molekulargewichtes. Schulz hat die Gl. (4) in den Grenzen $M=10^{4}-10^{6}$ geprüft. Wir wollen annehmen, daß sie mit den gleichen Zahlenwerten der Konstanten auch für höhere Molekulargewichte gilt, obwohl die entsprechende Behauptung bei Polyisobutylen und Polystyrol in Zweifel gezogen wird $^{7}$.

In Tab. 1 sind die Konzentration, die spezifische Viskosität, der Grenzwert $Z_{\eta}$ und der Polymerisationsgrad $P$ nach $\mathrm{K}$ uhn für die hier verwandten Fraktionen eingetragen. Außerdem ist noch der in der gleichen Weise bestimmte Polymerisationsgrad eines III. Ansatzes angegeben, der ähnlich hergestellt wie der Ansatz II, aber nicht fraktioniert, sondern nur einmal umgefällt war.

Der Fehler in der Bestimmung der Durchlauf-

${ }^{4}$ G. V. Schulz u. F. Blaschke, J. prakt. Chem. N. F. 158, 130 [1941].

s G. V. Schulz u. G. Sing, J. prakt. Chem. N. F. 161, 161 [1943]. zeiten betrug etwa $\pm 0,1$ Sek. bei Zeiten um 100

\begin{tabular}{|c|c|c|c|c|}
\hline Fraktion & $\begin{array}{c}c \\
\mathrm{~g} / l\end{array}$ & $\eta_{\mathrm{sp}}$ & $Z_{\eta}$ & $P$ \\
\hline \multirow[t]{3}{*}{ I } & 0,8167 & 1,202 & 1,091 & 38000 \\
\hline & 1,2066 & 1,710 & 0,947 & 32000 \\
\hline & 0,704 & 0,740 & 0,854 & 28000 \\
\hline B 3 & 1,056 & 1,168 & 0,826 & 27000 \\
\hline II A & 2,404 & 1,170 & 0,364 & 9800 \\
\hline 2 & 1,976 & 0,547 & 0,238 & 5800 \\
\hline В 3 & 2,656 & 0,491 & 0,162 & 3600 \\
\hline 4 & 4,292 & 0,524 & 0,106 & 2100 \\
\hline C 5 & 1,961 & 0,126 & 0,062 & 1100 \\
\hline \multirow{2}{*}{$\begin{array}{r}6 \\
\text { D } 7\end{array}$} & 2,240 & 0,0996 & 0,043 & 700 \\
\hline & 3,024 & 0,0966 & 0,031 & 470 \\
\hline III & 2,444 & 0,3997 & 0,149 & 3250 \\
\hline
\end{tabular}
Sek. und stieg bei längeren Durchlaufzeiten bis auf $\pm 0,2$ Sek., dementsprechend enthalten die Polymerisationsgrade von 500 und 30000 einen Fehler von $2 \%$ bzw. $0,5 \%$.

Tab. 1. Polymerisationsgrad $P$ der Fraktionen des Polymethacrylsäuremethylesters.

\section{B. Experimentelles}

Die Trübung wurde in der gleichen Weise bestimmt wie früher angegeben. Es erwies sich als zweckmäßig, in durchfallendem Licht zu beobachten, d. h. die im Thermostatenbad befindlichen Röhrchen mit einer Handlampe von unten her zu beleuchten. Besonders zur Messung bei ganz kleinen Molenbrüchen empfiehlt es sich, gleichzeitig ein Vergleichsröhrchen mit reinem Lösungsmittel zu beobachten. Die Abkühlungsgeschwindigkeit betrug etwa $1 / 10^{\circ}$ in 10 Sek., bei höherer Temperatur etwas mehr.

Im Einklang mit früheren Beobachtungen werden die Lösungen in Butanol und Propanol auch bei erhöhter Temperatur nicht vollständig klar, wenn sie mehr als etwa 10 Grundmolprozente Polymerisat enthalten. Das gilt sogar bei $135^{\circ} \mathrm{C}$, etwa $50^{\circ} \mathrm{C}$ über der Entmischungstemperatur. Dennoch läßt sich die Entmischung an einer plötzlichen Verstärkung der Trübung beim Abkühlen deutlich erkennen. Es dürfte sich um Lichtstreuung an den gelösten Kettenmolekülen handeln. Merkwürdig ist jedoch, daß in Cymol- und auch in Isopropanol ${ }^{8}$ eine solche Lichtstreuung nicht beobachtet wurde. In diesen Lösungsmitteln erhält man bei hinreichender Temperatur klare Lösungen.

Falsche Trübungstemperaturen werden beobachtet, wenn sich noch nicht eine homogene Lösung gebildet hat, sondern ein Teil des Polymerisats als zähe gequollene Masse am Glas klebt und so der übrigen Lösung entzogen ist.

${ }^{6}$ G. V. Schulz, Kolloid-Z. 115, 90 [1949].

' H. Mark, Mh. Chem. 81, 140 [1950].

8 E. Jenckel u. G. Coßmann, unveröffentlicht. 
C. Die gemessenen Löslichkeitskurven und kritischen Daten

Es wurde die Löslichkeit von Fraktionen des Polymethacrylesters in $p$-Cymol, $n$-Propanol und $n$ Butanol bestimmt, außerdem die Löslichkeit einer Fraktion in $\mathrm{C}_{6^{-}}$und $\mathrm{C}_{8^{-}}$-Alkohol. Die Lösungsmittel waren vorher sorgfältig entwässert worden und zwar die Alkohole mit frischem Kalk, $p$-Cymol mit

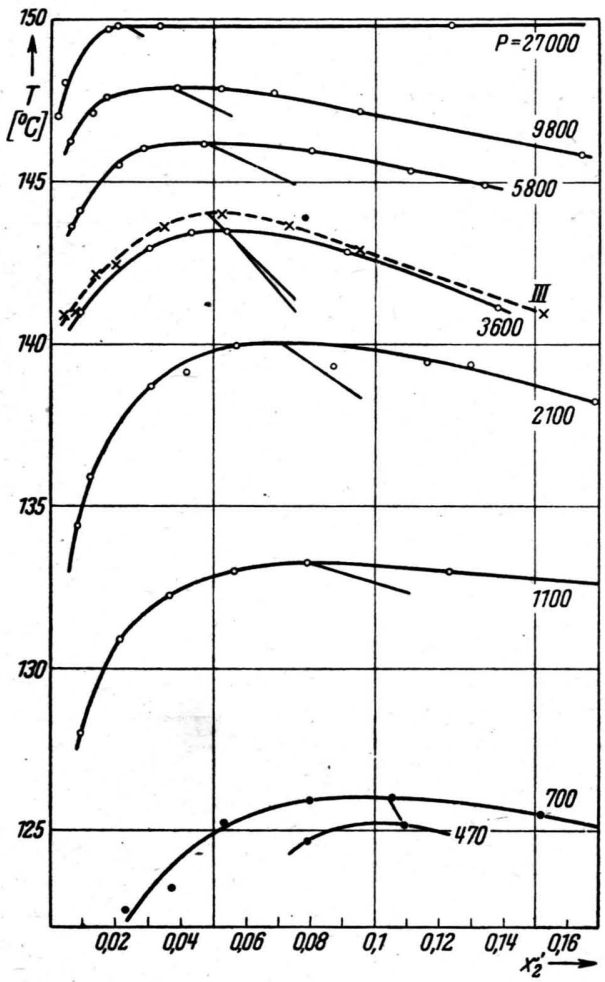

Abb. 1. Löslichkeitskurven von $p$-Cymol.

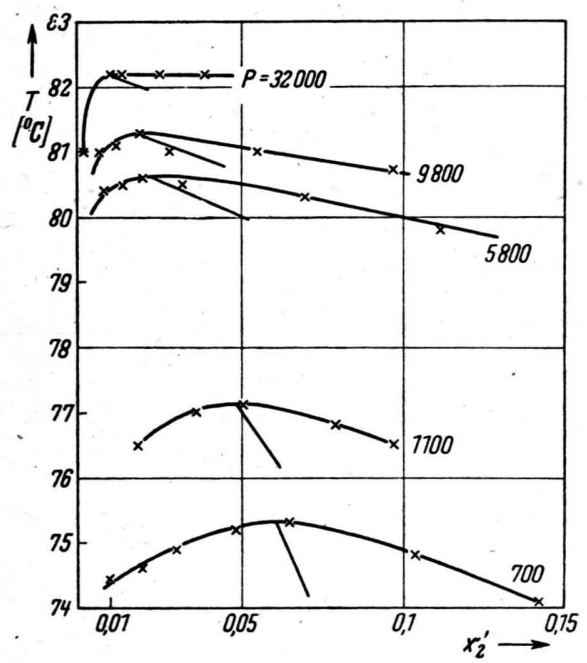

Abb. 2. Löslichkeitskurven von $n$-Propanol.
Chlorcalcium und Natriummetall. Der $\mathrm{C}_{8}$-Alkohol ist nicht getrocknet worden. Außer den Fraktionen wurde auch die Löslichkeit des unfraktionierten Ansatzes III bestimmt. Die Meßergebnisse sind in den Abb. 1-4 wiedergegeben. Die Konzentrationen sind als Grundmolenbrüche angegeben

$$
x_{1}^{\prime}=\frac{m_{1} / M_{1}}{m_{1} / M_{1}+m_{2} / M_{2}^{\prime}} \text { und } x_{2}^{\prime}=\frac{m_{2} / M_{2}^{\prime}}{m_{1} / M_{1}+m_{2} / M_{2}^{\prime}}
$$

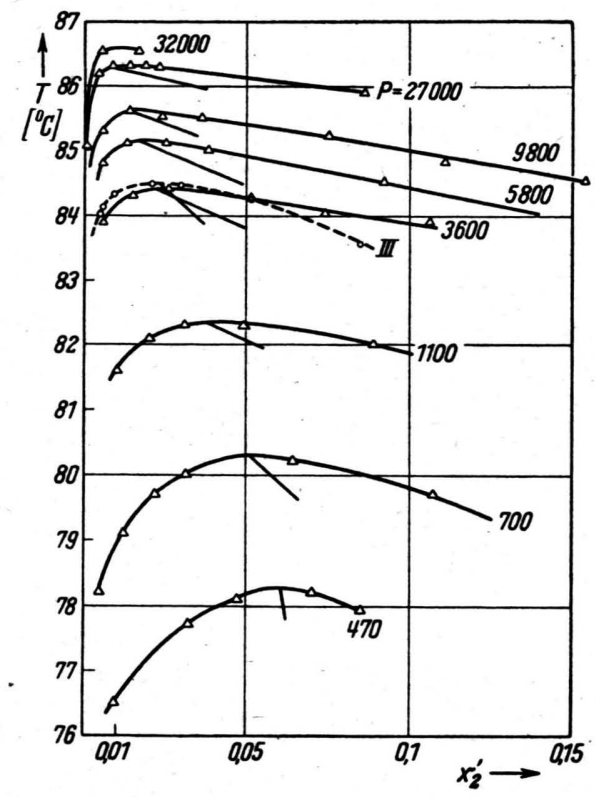

Abb. 3. Löslichkeitskurven von $n$-Butanol.

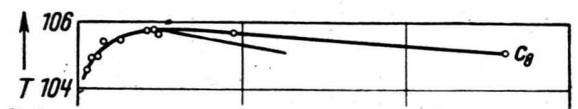

$\left.{ }^{\circ} \mathrm{C} C\right]$

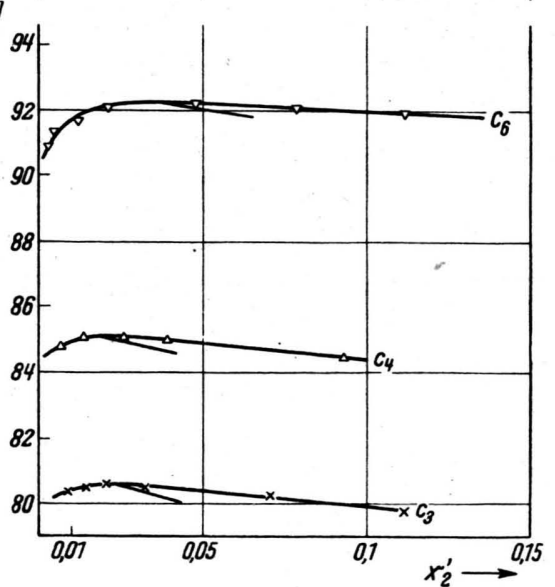

Abb. 4. Löslichkeitskurven der $\mathrm{C}_{3^{-}}, \mathrm{C}_{4^{-}}, \mathrm{C}_{6^{-}}, \mathrm{C}_{8^{-}}$Alkohole mit der Fraktion $P=5800$. 
$\left(m_{1}\right.$ und $m_{2}=$ Menge des Lösungsmittels bzw. des Polymerisates; $M_{1}$ und $\boldsymbol{M}_{2}^{\prime}=$ Molekulargewicht des Lösungsmittels bzw. des Grundmoleküls.)

Aus diesen Löslichkeitskurven kann man die kritische Temperatur sehr genau, die kritische Zusammensetzung aber sehr viel weniger genau entnehmen, weil die Kurven ein sehr flaches Maximum haben, besonders bei den Alkoholen, weniger beim in dem hier untersuchten Bereich der Kettenlängen die Zunahme etwa 4-mal größer. $T_{\text {krit }}$ nähert sich mit steigendem $P$ offenbar einem Grenzwert $T_{0}$ für unendlich lange Ketten.

Die kritische Zusammensetzung ist wie immer in den hochmolekularen Lösungen weit zur Seite des reinen Lösungsmittels hin verschoben und bewegt sich mit steigendem Polymerisationsgrad noch ein

\begin{tabular}{|c|c|c|c|c|c|c|c|c|c|}
\hline \multirow{3}{*}{$P$} & \multicolumn{3}{|c|}{$p$-Cymol } & \multicolumn{3}{|c|}{$n$-Propanol } & \multicolumn{3}{|c|}{$n$-Butanol } \\
\hline & \multirow{2}{*}{$\begin{array}{c}x_{2}^{\prime}{ }^{\prime} \text { krit } \\
\text { experim. }\end{array}$} & \multicolumn{2}{|c|}{ berechnet } & \multirow{2}{*}{$\begin{array}{c}x_{2}{ }^{\prime} \text { krit } \\
\text { experim. }\end{array}$} & \multicolumn{2}{|c|}{ berechnet } & \multirow{2}{*}{$\begin{array}{c}x_{2}{ }^{\prime} \text { krit } \\
\text { experim. }\end{array}$} & \multicolumn{2}{|c|}{ berechnet } \\
\hline & & Gl. (12) & $\Delta$ Gl. (26) & & Gl. (12) & $\Delta$ Gl. $(26)$ & & Gl. (12) & $\triangle$ Gl. $(26)$ \\
\hline 470 & - & 0,129 & $+0,042$ & - & 0,075 & $+0,010$ & 0,061 & 0,059 & $+0,007$ \\
\hline 700 & 0,104 & 0,110 & $+0,030$ & 0,061 & 0,062 & $+0,007$ & 0,051 & 0,049 & $+0,004$ \\
\hline 1100 & 0,080 & 0,091 & $+0,019$ & 0,049 & 0,050 & $+0,006$ & 0,039 & 0,040 & $+0,003$ \\
\hline 2100 & 0,071 & 0,068 & $+0,013$ & - & 0,037 & $+0,003$ & - & 0,029 & $+0,002$ \\
\hline 3600 & 0,054 & 0,054 & $+0,008$ & - & 0,029 & $+0,002$ & 0,022 & 0,022 & $+0,002$ \\
\hline 5800 & 0,048 & 0,045 & $+0,004$ & 0,023 & 0,023 & $+0,001$ & 0,018 & 0018 & $+0,001$ \\
\hline 9800 & 0038 & 0,035 & $+0,002$ & 0019 & 0,018 & $+0,001$ & 0,014 & 0,014 & \pm 0 \\
\hline 27000 & $(0,023)$ & 0,022 & $+0,001$ & - & 0,011 & \pm 0 & 0,010 & 0,085 & \pm 0 \\
\hline 32000 & - & 0,020 & \pm 0 & - & $\mathrm{C}, 010$ & \pm 0 & 0,009 & 0,008 & \pm 0 \\
\hline$\infty$ & - & 0 & 0 & - & 0 & 0 & - & 0 & 0 \\
\hline III & 0,049 & 0,055 & $+0,010$ & - & 0,030 & $+0,002$ & 0,024 & 0,024 & $+0,001$ \\
\hline
\end{tabular}

Tab. 2. Kritische Zusammensetzung als Funktion von $P$, experimentell und berechnet nach Gl. (12) und nach der Näherung (26). $\Lambda=\left[x_{2}{ }^{\prime}\right.$ krit nach Gl.(12) $-x_{2}{ }^{\prime}$ krit nach Gl. (26)].

\begin{tabular}{|c|c|c|c|c|c|c|c|c|c|}
\hline \multirow[b]{2}{*}{$\begin{array}{l}P \\
.\end{array}$} & \multicolumn{3}{|c|}{$p$-Cymol } & \multicolumn{3}{|c|}{$n$-Propanol } & \multicolumn{3}{|c|}{$n$-Butanol } \\
\hline & $T_{T_{\text {krit }}}^{\exp \mathrm{K}}$ & Gl. $(16)$ & Gl. ${ }_{\Delta}^{(32)}$ & $\underset{T_{\text {krit }}{ }^{0} \mathrm{~K}}{\exp }$ & Gl. ${ }_{\Delta}^{(16)}$ & ${ }_{\Delta}^{(G l . ~(32}$ & $\underset{T_{\text {krit }}{ }^{0} \mathrm{~K}}{\exp }$ & Gl. $(16)$ & Gl. $(32)$ \\
\hline 470 & 398,2 & $+0,9$ & $+7,6$ & - & - & - & 351,3 & $+1,3$ & - \\
\hline 700 & 399,1 & $\begin{array}{r}2,0 \\
+\quad 2,9\end{array}$ & $\begin{array}{r}9,0 \\
+9,7\end{array}$ & 348,3 & $+0,3$ & $+1,2$ & 353,2 & $\begin{array}{r}1,0 \\
+1,2\end{array}$ & $+1,7$ \\
\hline 1100 & 406,2 & $\begin{array}{r}0,7 \\
+0,7\end{array}$ & $\begin{array}{r}0,4 \\
+6,4\end{array}$ & 350,1 & $\begin{array}{r}0,0 \\
-0,4\end{array}$ & $\begin{array}{r}1,7 \\
+1,7\end{array}$ & 355,3 & $\begin{array}{r}1,5 \\
-0,5\end{array}$ & - \\
\hline 2100 & 413,0 & $-1,0$ & $\begin{array}{r}3,2 \\
+3,2\end{array}$ & - & - & - & - & $\begin{array}{r}0,0 \\
+0,2\end{array}$ & - \\
\hline 3600 & 416,4 & $-0,3$ & $\begin{array}{r}0,7 \\
+1,7\end{array}$ & - & - & - & 357,4 & $\begin{array}{l}0,2 \\
+0\end{array}$ & $+0,7$ \\
\hline 5800 & 419,1 & \pm 0 & $+0,6$ & 353,7 & $-0,4$ & \pm 0 & 358,1 & $+0,1$ & $+0,5$ \\
\hline 9800 & 420,8 & $+0,2$ & $\begin{array}{r}0,3 \\
+0,3\end{array}$ & 354,3 & +0.1 & $-0,1$ & 358,6 & $\begin{array}{r}0,6 \\
+0,6\end{array}$ & $\begin{array}{r}0,0 \\
+0,5\end{array}$ \\
\hline 27000 & 422,8 & $-0,3$ & $\begin{array}{r}+0,3 \\
+0\end{array}$ & - & - & - & 359,3 & $\begin{array}{r}0,3 \\
+0,3\end{array}$ & $+0,4$ \\
\hline 32000 & - & - & - & 355,2 & \pm 0 & $-0,2$ & 359,6 & $+0,1$ & $+0,2$ \\
\hline$\infty$ & \multicolumn{3}{|c|}{426,0 ber. mit Gl. (22) } & \multicolumn{3}{|c|}{356,0 ber. mit G1. (22) } & \multicolumn{3}{|c|}{360,6 ber. mit Gl. (22) } \\
\hline$P$ III & 417,0 & - & $+1,1$ & - & - & - & 357,5 & - & $+0,3$ \\
\hline
\end{tabular}

Tab. 3. Kritische Temperatur als Funktion von $P$, experimentell und berechnet nach Gl. (16) und nach der Näherung (32). $\Delta=T_{\text {ber }}-T_{\text {exp }}$.

Cymol. Zur leidlich genauen Bestimmung wurde die Regel von Cailletet und Mathias über den geraden Durchmesser angewendet. Die so gefundenen Werte von $T_{\text {krit }}$ und $x_{2 \mathrm{krit}}^{\prime}$ sind in Tab. 2-4 angegeben.

Mit steigendem Polymerisationsgrad nimmt die kritische Temperatur zu. Bei den Alkoholen ist diese Zunahme ziemlich gering, beim $p$-Cymol ist

\begin{tabular}{|l|l|l|}
\hline & $T_{\text {krit }}{ }^{0} \mathrm{~K}$ & $x_{2}{ }^{\prime}{ }^{\prime r i t}$ \\
\hline $\mathrm{C}_{3}$-Alkohol & 353,6 & 0,023 \\
$\mathrm{C}_{4}$-Alkohol & 358,1 & 0,017 \\
$\mathrm{C}_{6}$-Alkohol & 365,2 & 0,036 \\
$\mathrm{C}_{8}$-Alkohol & 378,8 & 0,026 \\
\hline
\end{tabular}

Tab. 4. Kritische Daten der $\mathrm{C}_{3^{-}}, \mathbf{C}_{4^{-}}, \mathbf{C}_{6}-, \mathbf{C}_{8}$-Alkohole mit der Fraktion $P=5800$. 
wenig weiter in der gleichen Richtung. Es sieht so aus, als ob die kritische Zusammensetzung für sehr lange Ketten gegen $x_{2 \mathrm{krit}}^{\prime}=0$ strebt.

Außer im Propanol und Butanol wurde die Löslichkeit auch in $\mathrm{C}_{6}$ - und $\mathrm{C}_{8}$-Alkohol bestimmt, jedoch nur mit der Fraktion $P=5800$. Die kritische Lösungstemperatur nimmt in dieser Reihe mit steigendem Kohlenstoffgehalt stark $\mathrm{zu}$, die Loslichkeit wird also schlechter. Da andererseits das Methanol sicher ein sehr schlechtes Losungsmittel ist, mit einer kritischen Temperatur, die viel höher liegt als die der hier untersuchten Alkohole, besteht offenbar ein Minimum der kritischen Temperatur oder ein Maximum der Löslichkeit beim Äthanol. Ein ähnliches Minimum der kritischen Temperatur wurde bei den entsprechenden Lösungen des Polystyrols beobachtet ${ }^{8 \mathrm{a}}$, jedoch nicht beim Äthanol, sondern erst beim $\mathrm{C}_{8^{-}}$bis $\mathrm{C}_{10^{-}}$-Alkohol. Auf den polaren, sauerstoffhaltigen Polymethacrylester wirkt schon ein kürzerer Paraffinrest löslichkeitsvermindernd als auf das unpolare Polystyrol. Methanol ist jedoch gegenüber beiden Polymerisaten ein sehr schlechtes Lösungsmittel, weil es zu ausgeprägt nur eine $\mathrm{Hy}$ droxylverbindung und daher chemisch zu unähnlich ist.

\section{Thermodynamische Berechnung}

1. Die Bedingungen für den kritischen Punkt

Zur thermodynamischen Berechnung gehen wir in der schon früher ${ }^{1}$ geübten Weise vor, indem wir die Formel

$$
\ln a_{1}=\frac{w_{1}}{R T}-\frac{s_{1}}{R}
$$

benutzen $\left(a_{1}=\right.$ Aktivität $=$ Dampfdruckverhältnis, $w_{1}=$ partielle Lösungswärme, $s_{1}=$ partielle Lösungsentropie). Da die Dampfdruckkurve bei der kritischen Temperatur und der kritischen Zusammensetzung einen Wendepunkt mit waagerechter Tangente, einen sog. Terrassenpunkt besitzt, lautet die Bedingung für den kritischen Punkt

$$
\frac{\mathrm{d} \ln a_{1}}{\mathrm{~d} x_{1}^{\prime}}=0 \text { und } \frac{\mathrm{d}^{2} \ln a_{1}}{\mathrm{~d} x_{1}^{\prime 2}}=0 .
$$

In der früheren Arbeit wurden verschiedene Formeln für die Entropie und die Lösungswärme bzw. die Aktivität hochmolekularer Lösungen nachge-

8a E. Jenckel u. G. Keller, Z. Naturforschg 5a, 317 [1950].

9 A. R. Miller, Proc. Cambridge philos. Soc. 38, 109 [1942]. prüft. Am besten bewährte sich damals eine Entropieformel nach Miller ${ }^{9}$, und fast gleichlautend nach $\mathrm{Huggins}{ }^{10}$ und Flory ${ }^{11}$

$s_{1}=-R\left[\ln x_{1}^{\prime}-\frac{z}{2} \ln \left(1-\frac{2}{z}\left(1-\frac{1}{P}\right) x_{2}^{\prime}\right)\right]$

in Verbindung mit folgendem Ausdruck für die Lösungswärme

$w_{1}=-v_{1} \Omega{x_{2}^{\prime}}^{2} ; \Omega=N_{\mathrm{L}}\left(\omega_{12}-\frac{\omega_{11}+\omega_{22}}{2}\right)$

( $z=$ Koordinationszahl, $v_{1}=$ Anzahl der van der Waals'schen Bindungen am Molekül des Lösungsmittels, gleichgesetzt derjenigen am Grundmolekül, also $v_{1} \approx z ; \omega_{12}, \omega_{11}, \omega_{22}=$ Energie einer van der Waals'schen Bindung zwischen einem Losungsmittelmolekül und einem Grundmolekül, bzw. zwischen zwei Lösungsmittelmolekülen bzw. zwei Grundmolekülen, $N_{\mathrm{L}}=$ Loschmidtsche Zahl). Daraus ergibt sich dann

$$
\begin{aligned}
\ln a_{1}=-\frac{v_{1} \Omega x_{2}^{\prime 2}}{R T} & +\ln x_{1}^{\prime} \\
& -\frac{z}{2} \ln \left(1-\frac{2}{z}\left(1-\frac{1}{P}\right) x_{2}^{\prime}\right) .
\end{aligned}
$$

Durch Differenzieren unter Beachtung von Gl. (2) erhalten wir, wenn wir noch die Abkürzungen $q=$ $1-1 / P, \quad r=1-2 / z$ und $x_{2 \mathrm{krit}}=x$ einführen und ferner $v_{1}=z$ setzen

$$
-\frac{\Omega}{R T} \frac{1}{1-r} 4 x=\frac{1}{1-x}-\frac{q}{[1-(1-r) q x]}
$$

und

$$
\begin{aligned}
& -\frac{\Omega}{R T} \frac{1}{1-r} 4 x \\
& =\frac{q^{2}(1-r)-1+2 q x(1-r)(1-q)+r(1-r) q^{2} x^{2}}{\left[1-(1+q-r q) x+(1-r) q x^{2}\right]^{2}} .
\end{aligned}
$$

2. Berechnung der kritischen Zusammensetzung

Dividiert man Gl. (10) und (11) durcheinander, so erhält man folgende Gleichung dritten Grades, in der $x$ nur eine Funktion von $q$ und $r$, also von $P$ und $z$ ist, aber nicht von $\Omega$.

$$
\begin{aligned}
x^{3}[ & \left.2 r q^{2}(1-r)\right]+x^{2}[r q(r q+2 q-4)-3 q(q-1)] \\
& +x 2\left[r q-1+q^{2}(1-r)\right]+(1-q)=0 .
\end{aligned}
$$

10 M. L. Huggins, Ann. New York Acad. Sci. 43, 1 [1942].

${ }_{11}$ P. J. Flory, J. chem. Physics 10, 51 [1942]; 9, 160 [1941]. 


\begin{tabular}{|c|c|c|c|c|c|c|c|c|}
\hline & $z$ & $P=10$ & 50 & 500 & 5000 & 2500 & 5000 & $\infty$ \\
\hline 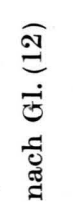 & $\begin{array}{l}2 \\
2,05 \\
2,1 \\
2,4 \\
2,7 \\
3,0 \\
4,0\end{array}$ & $\begin{array}{l}0,350 \\
0,337 \\
0,327 \\
0,292 \\
0,279 \\
0,270 \\
0,260\end{array}$ & $\begin{array}{l}0,337 \\
0,276 \\
0,248 \\
0,183 \\
0,162 \\
0,150 \\
0,137\end{array}$ & $\begin{array}{l}0,334 \\
0,147 \\
0,117 \\
0,071 \\
0,060 \\
0,055 \\
0,049\end{array}$ & $\begin{array}{l}0,334 \\
0,057 \\
0,043 \\
0,024 \\
0,020 \\
0,019 \\
0,016\end{array}$ & $\begin{array}{l}0,333 \\
0,028 \\
0,020 \\
0,011 \\
0,009 \\
0,008 \\
0,007\end{array}$ & $\begin{array}{l}0,333 \\
0,021 \\
0,015 \\
0,008 \\
0,007 \\
0,006 \\
0,005\end{array}$ & $\begin{array}{l}0,333 \\
0,0 \\
0,0 \\
0,0 \\
0,0 \\
0,0 \\
0,0\end{array}$ \\
\hline 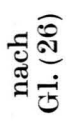 & $\begin{array}{l}2,05 \\
2,1 \\
2,4 \\
3,0\end{array}$ & $\overline{-}$ & $\begin{array}{l}0,329 \\
0,237 \\
0,131 \\
0,097\end{array}$ & $\begin{array}{l}0,20 \\
0,146 \\
0,080 \\
0,060\end{array}$ & $\begin{array}{l}0,064 \\
0,046 \\
0,025 \\
0,019\end{array}$ & $\begin{array}{l}0,029 \\
0,021 \\
0,011 \\
0,008\end{array}$ & $\begin{array}{l}0,020 \\
0,015 \\
0,008 \\
0,006\end{array}$ & - \\
\hline
\end{tabular}

Tab. 5. Berechnete Werte der kritischen Zusammensetzung $x=(P, z)$ für vorgegebene runde Werte $P$ und $z$ nach Gl. (12) und nach der Näherung (26).

Die nach dem Verfahren von Horner berechneten Lösungen dieser Gleichung sind für runde Zahlen $z$ und $P$ in Tab. 5 angeführt. Für $z=2$ strebt $x$ mit wachsendem $P$ rasch gegen $1 / 3$. Für $P=\infty$ wird $x=0$ für alle $z$ außer $z=2$. (Die Tab. 5 ist wesentlich ausführlicher und genauer als die früher mitgeteilte.) In Abb. 5' sind die berechneten Werte von $\log x$ gegen $\log P$ mit dem Parameter $z$ aufgetragen; sie stellen leicht gekrümmte Kurven dar. Außerdem sind aber die experimentell gemessenen Werte $x$ ein-

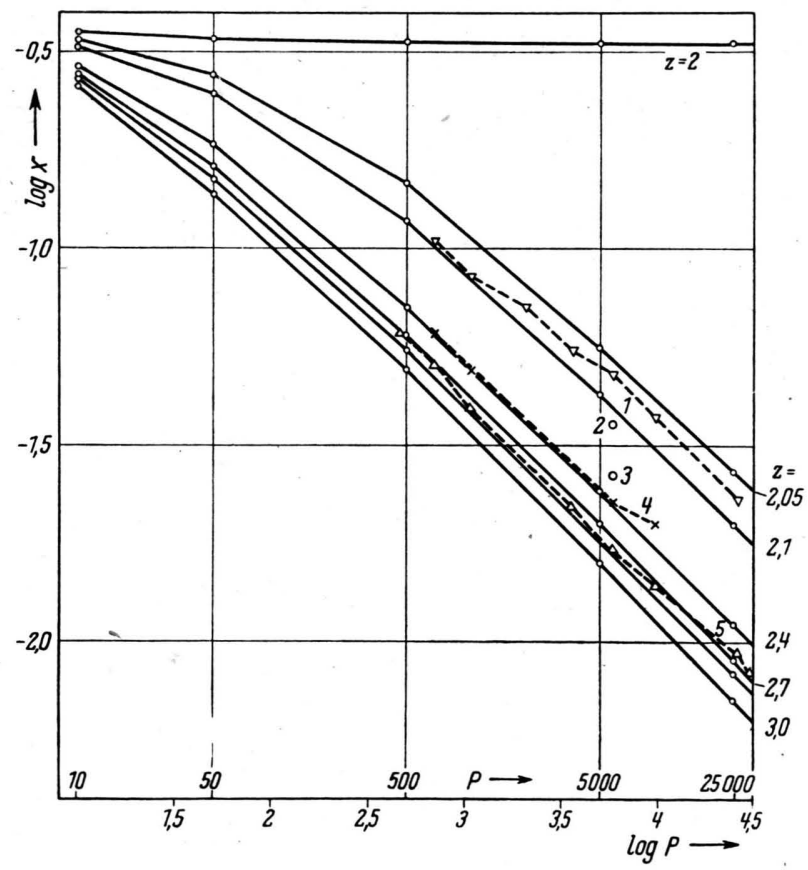

Abb. 5. Änderung der krit. Zusammensetzung $x$ mit dem Polymerisationsgrad $P$ für verschiedene Koordinationszahlen $z$. Ausgezogene Kurven berechnet nach Gl. (12). Gestrichelte Kurven verbinden Meßpunkte. $1=p$-Cymol $\nabla \nabla, 2=\mathrm{C}_{6}$-Alkohol $\circ \circ, 3=\mathrm{C}_{8}$-Alkohol ००, $4=n$-Propanol $\times \times, 5=n$-Butanol $\Delta \Delta$. getragen, die, wie man sieht, sich verhältnismäßig gut einfügen, zumal wenn man die experimentellen Fehler bei der Bestimmung von $x$ in Rechnung setzt. Damit ist gezeigt, daß die verwendeten Ansätze (7) und (8) die kritische Zusammensetzung richtig wiedergeben.

Umgekehrt läßt sich aus Tab. 5 auch die Koordinationszahl $z$ einer Lösung bestimmen, wenn $P$ und $x$ bekannt sind. Zweckmäßig zeichnet man Abb. 5 so um, daß der im Versuch vorgegebene $P$-Wert den Parameter bildet und $\log x$ und $\log (z-2)$ die Koordinaten darstellen. In dieser Darstellung (Abb. 6) ergeben sich, im Gegensatz zu der Auftragung gegen $\log z$ fast geradlinige, zur Interpolation sehr geeignete Kurven. Außerdem enthält Abb. 6 die gemessenen Werte von $x$. Sie sollten jeweils senkrecht

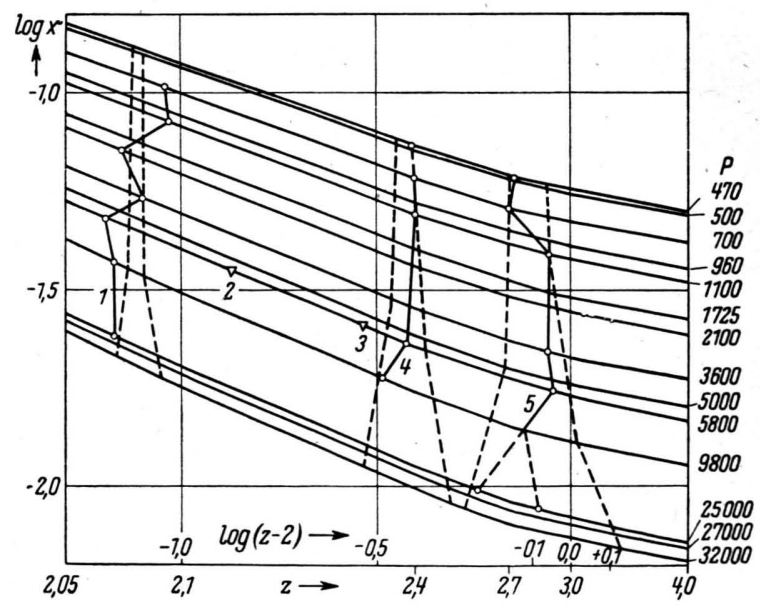

Abb. 6. Änderung der krit. Zusammensetzung $x$ mit der Koordinationszahl $z$ für verschiedene Polymerisationsgrade $P$. Ausgezogene Kurven nach Gl. (12) berechnet. Gestrichelter Bereich: Einfluß eines Fehlers von 0,001 in $x$ auf $z$. Meßwerte: $1=p$-Cymol, $2=$ $\mathrm{C}_{6}$-Alkohol, $3=\mathrm{C}_{8}$-Alkohol, $4=n$-Propanol, $5=n$ Butanol. 
übereinander stehen und damit einen bestimmten Wert von $z$ angeben. Genau ist das nicht der Fall, weil der experimentelle Fehler in $x$ sich stark bemerkbar macht. In Abb. 6 ist gestrichelt angedeutet, in welchem Bereich sich die Werte log $(z-2)$ verschieben würden, wenn man einen Fehler in $x$ von $\pm 0,001$ annimmt; diesen Fehler muß man unbedingt zulassen. Immerhin läßt sich doch ein $z$-Wert ableiten, wenn man von den Messungen mit den Polymerisaten $P=27000$ und $P=32000$ absieht, deren Werte $x$ wegen des besonders flachen Verlaufs der Löslichkeitskurve ungenauer sind. Die so ermittelten Werte $z$ sind mit ihrem Fehler in Tab. 6 zusammengestellt; sie erscheinen gegenüber den früher angegebenen hauptsächlich deswegen verändert, weil die Kettenlänge $P$ nach einer anderen Gesetzmäßigkeit (Gl.4) aus der spezifischen Viskosität errechnet wurde. Mit den so ermittelten $z$ Werten und den bekannten $P$-Werten kann man wiederum nach Gl. (12) oder zweckmäßiger durch Interpolation in Tab. 5 die zugehörigen $x$-Werte berechnen. Sie sind in Tab. 2 angegeben.

\begin{tabular}{|l|l|c|c|}
\hline Lösungsmittel & \multicolumn{1}{|c|}{$z$-Wert } & $-\alpha$ & $\beta$ \\
\hline p-Cymol & $2,077 \pm 0,015$ & 0,230 & 105,8 \\
$n$-Propanol & $2,375 \pm 0,015$ & 1,003 & 385,3 \\
$n$-Butanol & $2,8 \pm 0,1$ & 1,498 & 591,5 \\
C $_{6}$-Alkohol & $(2,13)$ & - & - \\
C $_{8}$-Alkohol & $(2,29)$ & - & - \\
Chloroform & unter 2,05* nicht ablesbar & \\
Toluol & untol \\
\hline
\end{tabular}

* nach den Messungen von Jenckel und Gorke ${ }^{1}$ und Berechnung von $P$ nach $K u h n$ mit Gl. (4).

Tab. 6. Koordinationszahlen $z$ und $\alpha$ - und $\beta$-Werte in verschiedenen Lösungsmitteln.

3. Berechnung der kritischen Temperatur mit Gl. (13)

Zur Berechnung der krit. Temperatur $T_{\text {krit }}$ braucht man nur Gl. (10) nach $T$ aufzulösen. Man erhält

$$
\begin{array}{r}
T_{\text {krit }}=-\frac{\Omega}{R}\left[4 x \frac{1-\left(1+q(1-r) x+q(1-r) x^{2}\right.}{(1-r)(1-q+r q x)}\right] \\
=-\frac{\Omega}{R} \frac{1}{A}, \quad
\end{array}
$$

wenn wir den Klammerausdruck gleich $\mathbf{l} / A$ setzen. Die Größe $A$ ist also eine Funktion von $r=f(z)$ und von $q=f(P)$ und weiter von $x=f(z, P)$, insgesamt also nur eine Funktion von $z$ und $P$. Die in Tab. 7 aufgeführten Werte $\log A$ sind für vorgegebene Werte $z$ und $P$ berechnet. Durch Interpolation kann man die $A$-Werte ermitteln, die zu den in

\begin{tabular}{|l|c|c|c|c|c|}
\hline \multicolumn{1}{|c|}{$z$} & $P=10$ & 50 & 500 & 5000 & 2500 \\
\hline 2 & $-0,7947$ & $-1,4761$ & $-2,4722$ & $-3,4718$ & $-4,1708$ \\
2,05 & $-0,7785$ & $-1,3551$ & $-1,8904$ & $-2,1153$ & $-2,1757$ \\
2,1 & $-0,7662$ & $-1,2802$ & $-1,7078$ & $-1,8682$ & $-1,9107$ \\
2,4 & $-0,7316$ & $-1,0878$ & $-1,3342$ & $-1,4192$ & $-1,4438$ \\
2,7 & $-0,7290$ & $-1,0244$ & $-1,2204$ & $-1,2871$ & $-1,3044$ \\
3,0 & $-0,7384$ & $-1,0001$ & $-1,1703$ & $-1,2280$ & $-1,2431$ \\
4,0 & $-0,7967$ & $-1,0055$ & $-1,1383$ & $-1,1828$ & $-1,1946$ \\
\hline
\end{tabular}

Tab. 7. Berechnete Werte von $\log A=f(P, z)$ vgl. Gl. (13).

dieser Arbeit vorkommenden $P$ - und $z$-Werten gehören. Zu diesem Zwecke trägt man $\log P$ gegen $\log A$ mit dem Parameter $z$ auf. Aus dieser Darstellung läßt sich $\log A$ für die experimentellen $P$. Werte, aber nur für die vorgegebenen runden $z$ Werte entnehmen. In einer zweiten Darstellung trägt man jetzt diese $\log A$ zweckmäßig gegen $\log (z-2)$ mit dem Parameter der experimentellen $P$-Werte auf und kann daraus $\log A$ für die experimentellen $P$ - und $z$-Werte entnehmen. Sie sind hier nicht aufgeführt, ergeben sich aber aus den $\mu$-Werten der Tab. 8 durch Division mit $z$.

\begin{tabular}{|r|r|r|r|}
\hline \multicolumn{1}{|c|}{$P$} & $p$-Cymol & $n$-Propanol & $n$-Butanol \\
\hline 470 & $3,53 \cdot 10^{-2}$ & $10,73 \cdot 10^{-2}$ & $17,98 \cdot 10^{-2}$ \\
700 & $3,24 \cdot 10^{-2}$ & $10,21 \cdot 10^{-2}$ & $17,08 \cdot 10^{-2}$ \\
1100 & $2,99 \cdot 10^{-2}$ & $9,88 \cdot 10^{-2}$ & $16,94 \cdot 10^{-2}$ \\
2100 & $2,64 \cdot 10^{-2}$ & $9,31 \cdot 10^{-2}$ & $16,18 \cdot 10^{-2}$ \\
3600 & $2,39 \cdot 10^{-2}$ & $8,83 \cdot 10^{-2}$ & $15,74 \cdot 10^{-2}$ \\
5800 & $2,20 \cdot 10^{-2}$ & $8,74 \cdot 10^{-2}$ & $15,35 \cdot 10^{-2}$ \\
9800 & $2,10 \cdot 10^{-2}$ & $8,43 \cdot 10^{-2}$ & $14,90 \cdot 10^{-2}$ \\
27000 & $2,015 \cdot 10^{-2}$ & $8,19 \cdot 10^{-2}$ & $14,70 \cdot 10^{-2}$ \\
\hline
\end{tabular}

Tab. 8. $\mu$-Werte für die hier benutzten Fraktionen und Lösungsmittel.

Man muß nun annehmen, daß $\Omega$, welches ja nur die Differenz der Bindungsenergie ungleichartiger Partner gegenüber dem Mittelwert derjenigen gleichartiger Partner darstellt, vom Polymerisationsgrad $P$ unabhängig ist. Dann aber läßt sich, wie schon früher gefunden und an dieser Stelle zu bestätigen ist, Gl. (13) zur Darstellung von $T_{\text {krit }}$ als Funktion von $P$ nicht mit der Beobachtung in Einklang bringen. Wählt man beispielsweise $\Omega$ so, daß für $P=9800$ die berechnete und beobachtete Temperatur übereinstimmen, so liegt für $P=700$ die berechnete Temperatur bei $p$-Cymol um $124^{\circ}$, bei $n$ Butanol um $48^{\circ}$ und bei $n$-Propanol um $56^{\circ} \mathrm{zu}$ tief. Im gleichen Intervall von $P$ nimmt im Versuch $T_{\text {krit }}$ nur um $22^{0}$ bzw. $5^{0}$ bzw. $6^{0}$ ab.

Die Diskrepanz zwischen Beobachtung und Theorie nach Gl. (13) tritt in dieser Arbeit noch stärker 
als früher zutage, weil die Kettenlängen über einen wesentlich größeren Bereich verstreut liegen. Außerdem wurde, wenigstens für Butanol, ein beträchtlich kleinerer $z$-Wert gefunden, der ebenfalls bewirkt, daß $T_{\text {krit }}$ mit $P$ nach Gl. (13) stärker ansteigt.

Früher wurde die schlechte Übereinstimmung auf einen experimentellen Fehler, nämlich die mangelhafte Einheitlichkeit der Fraktionen zurückgeführt. Sie sollte bewirken, daß in den kurzkettigen Fraktionen der Anteil an langen Ketten zu früh ausfällt und so eine zu hohe Trübungstemperatur vortäuscht. Das erscheint jedoch jetzt, angesichts der relativ kleinen Abnahmen von $P$, die man beobachtet, und der riesigen Differenzen mit der Rechnung doch als ausgeschlossen. Obendrein beweisen die Versuche mit unfraktioniertem Material (Ansatz III), deren kritische Temperatur um höchstens $1^{0}$ tiefer liegt als die Fraktion gleicher mittlerer Kettenlänge, daß der Einfluß der Unheitlichkeit überhaupt nur gering sein kann.

\section{Berechnung der kritischen Temperatur nach Gl. (16)}

In der Literatur findet man statt Gl. (9) auch den Ausdruck

$$
\begin{gathered}
\ln a_{1}=\ln x_{1}^{\prime}+\left(1-\frac{1}{P}\right) x_{2}^{\prime}+\mu x_{2}^{\prime 2}, \\
\text { worin } \mu=\alpha+\frac{\beta}{T} \text { ist. }
\end{gathered}
$$

Die Formel geht auf Huggins ${ }^{12}$ und Flory ${ }^{11} \mathrm{zu}$ rück.

Sie führt, wie schon früher gezeigt ${ }^{1}$ wurde, angewandt auf die Entmischung zu falschen Ergebnissen, denn zur Berechnung von $x_{2}{ }^{\prime}$ krit wird $\mu$ wieder herausdividiert und es sollte daher $x$ unabhängig vom Lösungsmittel sein, was offensichtlich nicht stimmt. Die so berechneten $x$-Werte liegen außerdem viel zu tief.

Es lag aber nahe, die Gl. (9) und (14) zu kombinieren, also $z$ und $\mu$ nebeneinander zu verwenden, was zu folgendem Ausdruck führt

$$
\ln a_{1}=\ln x_{1}^{\prime}-\frac{z}{2} \ln \left[1-\frac{2}{z}\left(1-\frac{1}{P}\right) x_{2}^{\prime}\right]+\mu x_{2}^{\prime 2} \text {. }
$$

Die Bedingungen (6) lauten dann $\left(x_{2 \mathrm{krit}}^{\prime}=x\right)$

$$
2 \mu x=\frac{1}{1-x}-\frac{q}{[1-(1-r) q x]},
$$

12 M. L. Huggins, J. Amer. Chem. Soc. 64, 1712 [1942]; J. chẹm. Physics 9, 440 [1941]; J. physic. Chem. 46, 151 [1942].
$2 \mu=\frac{q^{2}(1-r)-1+2 q x(1-r)(1-q)+r(1-r) q^{2} x^{2}}{\left[1-(1+q-r q) x+(1-r) q x^{2}\right]^{2}}$.

Zur Berechnung von $x$ dividiert man wieder (17) und (18) und erhält dieselbe Gleichung (13) dritten Grades wie oben. Es resultieren daher gleiche $z$ und gleiche $x$ wie in Tab. 5 und 6 .

Zur Berechnung von $T_{\text {krit }}$ löst man (17) nach $\mu$ auf; man erhält

$$
\mu=\frac{1-q+r q x}{1-(1+q-r q) x+(1-r) q x^{2}} \frac{1}{2 x} .
$$

Dieser Ausdruck läßt sich leicht auf die oben schon verwendete Größe $A$ zurückführen

$$
\mu=A \frac{2}{1-r}=A z \text {. }
$$

Bei Gl. (19) und (20) sei daran erinnert, daß $x$ und $A$ nur Funktionen von $q$ und $r$, also von $P$ und $z$ sind. Die Werte von $A$ für die hier vorkommenden Polymerisationsgrade und Koordinationszahlen (letztere bestimmt aus experimentellen $x$-Werten) lassen sich aus Tab. 7 interpolieren, wie oben angegeben. Es sind daher einfach nach Gl. (20) die entsprechenden $\mu$-Werte auszurechnen, die in Tab. 8 verzeichnet sind. Diese $\mu$-Werte sollten gemäß Gl. (15) sich linear mit der reziproken Temperatur ändern. Das ist in
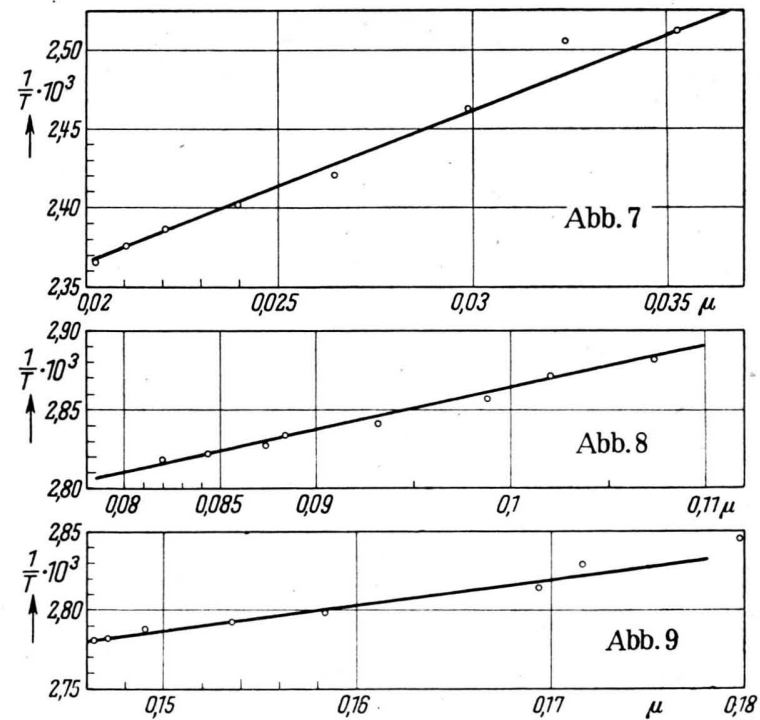

Abb. 7. Änderung von $\mu=\alpha+\beta / T$ mit der reziproken Temperatur bei $p$-Cymol.

Abb. 8. Änderung von $\mu=\alpha+\beta / T$ mit der reziproken Temperatur bei $n$-Propanol.

Abb. 9. Änderung von $\mu=\alpha+\beta / T$ mit der reziproken Temperatur bei $n$-Butanol. 
der Tat der Fall, wie Abb. 7-9 zeigen, aus denen man die Konstanten $\alpha$ und $\beta$ mit den in Tab. 6 wiedergegebenen Zahlenwerten entnehmen kann.

Mit diesen $\alpha$ - und $\beta$-Werten berechnen sich nach Gl. (15) Werte der krit. Temperatur, die in Tab. 3 den experimentellen gegenübergestellt sind. Die Übereinstimmung der Zahlen ist ausgezeichnet. Gl. (16) gibt also im Gegensatz zu Gl. (9) die Versuchsergebnisse vollkommen richtig wieder ${ }^{13}$.

Schließlich mögen noch die Werte $\mu=\mu_{0}$ und $T_{\text {krit }}=T_{0}$ für unendlich lange Ketten $P=\infty$, $q=1$ betrachtet werden. Wenn man beachtet, daß sich in diesem Falle $x$ im Zähler und Nenner kürzt und weiterhin $x=0$ wird, so erhält man aus Gl. (19)

$$
\mu_{0}=\frac{r}{2}=\frac{1}{2}-\frac{1}{z}
$$

und nach Gl. (15)

$\frac{1}{T_{0}}=-\frac{\alpha}{\beta}+\frac{r}{2 \beta}=-\frac{\alpha}{\beta}+\frac{1}{\beta}\left(\frac{1}{2}-\frac{1}{z}\right)$.

Tab. 3 enthält die so berechneten Werte $T_{0}$. Sie stimmen überein mit den aus einer Darstellung $1 / T_{\text {krit }}$ gegen $1 / P$ extrapolierten experimentellen Werten $T_{0}$.

\section{Näherungsformeln}

Die oben abgeleiteten Formeln sind etwas unhandlich. Es lassen sich jedoch gute Näherungen angeben.

a) Näherungen für $x=f(P, z)$

Setzt man in Gl. (12) $q \approx 1$, so erhält man

$$
\begin{aligned}
x^{3} 2 r(1-r)+x^{2}[r(r-2)- & 3(q-1)] \\
& +(1-q)=0 .
\end{aligned}
$$

Diese Gleichung gibt für hohe Polymerisationsgrade sehr genaue Werte; z. B. erhält man für $z=3$ und $P=5000$ den Wert $x=0,0187$ gegenüber $x=$ 0,0185 in Tab. 5. Wegen des kubischen Gliedes ist Gl. (23) jedoch noch recht unbequem.

Als weitere Näherung kann man das kubische Glied vernachlässigen. Man erhält

$$
\begin{gathered}
x^{2}[r(r-2)-3(q-1)]+(1-q)=0, \\
x=\sqrt{\frac{1}{\operatorname{Pr}(2-r)-3}} .
\end{gathered}
$$

Läßt man schließlich noch -3 im Nenner fort, da es meist klein ist gegenüber $\operatorname{Pr}(2-r)$, so resultiert

13 Es gelang jedoch bisher nicht, die ganze Löslichkeitskurve befriedigend zu berechnen.

$$
x=\frac{1}{\sqrt{P}} \frac{1}{\sqrt{1-4 / z^{2}}} .
$$

Gl. (26) gibt eine sehr einfache Beziehung zwischen $x$ und $P$ an, die, wie man sehen wird, trotz der vielen Näherungen recht brauchbar ist. Die nach Gl. (26) für gegebenes $P$ und $z$ berechneten $x$-Werte sind in Tab. 5 angeführt. Zur Prüfung der Güte der Näherung vergleiche man sie mit den dort ebenfalls angeführten exakten Zahlen nach Gl. (12).

Bei Münster ${ }^{14}$ findet man die Form

$$
x=\frac{1}{\sqrt{P}} \frac{1}{\sqrt{2-8 / z^{2}}} .
$$

Sie geht ebenfalls auf die Millersche Formel für die athermische Mischungsentropie zurück und wurde durch Reihenentwicklungen erhalten. Sie stimmt mit der Näherung (26) bis auf einen Faktor $1 / \sqrt{2}$ überein. Wenn man jedoch dieWerte der Tab. 5, die sich aus Gl. (26) ergeben, mit diesem Faktor $1 / \sqrt{2}$ multipliziert, so resultieren Zahlen, die nicht unbeträchtlich von den exakten abweichen. Jedenfalls liefert Gl. (26) bessere Werte als Gl. (26a).

$\beta$ ) Näherungen für $T_{\text {krit }}=f(P, z, \alpha, \beta)$

Man kann in Gl. (19) $q \approx 1 ; x \ll 1$ einführen und erhält dann

$$
\begin{aligned}
\mu & =\frac{1-q}{2 x[1-(2-r) x]}+\frac{r}{2[1-(2-r) x]} \\
& \approx \frac{1-q}{2 x}[1+(2-r) x]+\frac{r}{2}[1+(2-r) x] \\
& \approx \frac{1-q}{2 x}+\frac{r}{2}+\frac{r}{2}(2-r) x .
\end{aligned}
$$

Gl. (29) liefert $\mu$-Werte, die mit den nach Gl. (20) exakt berechneten gut übereinstimmen, was hier aber nicht weiter gezeigt werden soll.

Aus Gl. (29) erhält man sogleich in Verbindung mit Gl. (26)

$$
\mu=\sqrt{\frac{r(2-r)}{P}}+\frac{r}{2}
$$

oder mit Gl. (15)

$$
\frac{1}{T_{\text {krit }}}=-\frac{\alpha}{\beta}+\frac{1}{\beta}\left[\frac{r}{2}+\sqrt{\frac{r(2-r)}{P}}\right]
$$

und mit Gl. (22)

$$
\begin{aligned}
\frac{1}{T_{\mathrm{krit}}}=\frac{1}{T_{0}}+\frac{1}{\beta} \sqrt{\frac{r(2-r)}{P}} & =\frac{1}{T_{0}} \\
& +\frac{1}{\beta} \sqrt{\frac{1-4 / z^{2}}{P}} .
\end{aligned}
$$

14 A. Münster, Kolloid-Z. 120, 141 [1951]. 
Gl. (32) ist gegenüber den ursprünglichen Gleichungen an Einfachheit kaum zu überbieten. Trotz der sehr vielen Näherungen liefert sie Werte, die recht gut mit den experimentellen und den exakt berechneten der Gl. (16) zusammenpassen, wie die Zusammenstellung in Tab. 3 zeigt.

Die Näherungen (26) und (32) erlauben es, rasch und ziemlich sicher die kritischen Koordinaten zu berechnen. Danach ändert sich $x_{\text {krit }}$ proportional und $1 / T_{\text {krit }}$ linear mit $1 / \sqrt{P}$.

6. Einige Bemerkungen über $\mu$ und $z$

Der Wert $\mu$ setzt sich nach Gl. (15)

$$
\mu=\alpha+\frac{\beta}{T}
$$

15 Das Verhältnis $\alpha / \beta$ läßt sich vielleicht zufällig bei den Stoffen der Tab. 6 recht gut durch die Beziehung $\alpha / \beta=$ const $\cdot \ln M_{1}$ wiedergeben $\left(M_{1}=\right.$ Molekulargewicht des Lösungsmittels). DerWert der Konstanten ergibt sich bei Cymol, Propanol und Butanol zu 1,07 bzw. 1,07 bzw. $1,09 \cdot 10^{-2}$.

Merkwürdigerweise nimmt auch die sogenannte Koordinationszahl $z$ mit steigendem $\alpha$ oder $\beta$ zu, wenigstens in den drei hier genauer untersuchten Lösungen. Es gelten ungefähr folgende Beziehungen:

$$
\alpha=a(z-2)^{3 / 4} ; \beta=b(z-2)^{3 / 4} .
$$

aus $\alpha$ und $\beta$ zusammen. Führt man Gl. (15) in (14) oder (16) ein und vergleicht man mit (5), so erkennt man sogleich, daß $\beta$ einen Ausdruck für die Lösungswärme, $\alpha$ dagegen einen Entropieausdruck bedeutet. Die Zahlenwerte von $\alpha$ und $\beta$ laufen nun, wie Tab. 6 klar erkennen läßt, einander ungefähr prọportional. Da der Millersche Ausdruck (7) die Mischungsentropie einer athermischen Lösung wiedergibt, dürfte $\alpha$ den zusätzlichen Entropieanteil darstellen, der wegen der bevorzugten Bildung von Assoziaten in schlechten Lösungsmitteln (oder von Solvaten in guten Lösungsmitteln, was hier jedoch nicht interessiert) auftritt. Daher wird $\alpha$ größer in $\operatorname{dem}$ Maße wie auch $\beta$ größer wird $^{15}$.

Da.im Kettenmolekül zwei Koordinationsstellen eines Grundmoleküls auf jeden Fall durch Grundmoleküle eingenommen sind, bedeutet $(z-2)$ die Zahl der Lösungsmittelmoleküle, die weiterhin benachbart sein können. Nur die letzteren tragen zur Lösungswärme bei und daher könnte $\beta$ symbat mit $(z-2)$ anwachsen. $\mathrm{Ob}$ man allerdings überhaupt noch anschaulich von ,Koordination“" sprechen darf, wenn $(z-2)$ den sehr kleinen Wert von 0,07 ausmacht, wie beim Cymol, mag dahingestellt sein.

\title{
Ein Thermostat zur Erreichung von $50^{\circ}$ abs ohne Benutzung von flüssigem Wasserstoff
}

\author{
Von Eduard Justi
}

Aus dem Institut für technische Physik der Technischen Hochschule Braunschweig

(Z. Naturforschg. 7 a, 692-699 [1952]; eingegangen am 24. Juli 1952)

Herrn Prof. Dr. Walther Meissner zum 70. Geburtstag am 16. Dezember 1952 in dankbarer Verehrung gewidmet

\begin{abstract}
Während die Tripelpunktstemperatur von reinem $\mathrm{N}_{2} 63^{\circ}$ abs und die von reinem $\mathrm{O}_{2}$ $54^{0}$ abs beträgt, bildet Luft mit ca. 22 Vol.- $\% \mathrm{~N}_{2}$ ein Eutektikum, das bei $50,1^{\circ}$ abs mit anomal hoher Wärmekapazität erstarrt. Der Umstand, daß gewöhnliche flüssige Luft zufällig etwa diese Zusammensetzung aufweist, wird hier zur Konstruktion eines Ganzmetall-Thermostaten für den Temperaturbereich $88^{\circ}-50^{\circ}$ abs ausgenutzt. Für Institute ohne $\mathrm{H}_{2}$-Verflüssigungsanlage kann diese Minimaltemperatur vielfach ein $\mathrm{H}_{2}$-Bad ersetzen. Dies liegt nicht nur daran, daß die absolute Endtemperatur ca. $50 \%$ unter dem Siedepunkt der Luft liegt, sondern ist besonders darin begründet, daß viele Eigenschaften der Materie, wie z. B. die spezifische Wärme der Festkörper oder der elektrische Widerstand von Metallen und die Leitfähigkeit von Halbleitern in diesem Bereich beschleunigt mit $T$ abfallen. Als praktisches Beispiel hierfür wird gezeigt, wie die altbekannte Reinheitsprüfung von Metallen nach der Restwiderstandsmethode durch Anwendung des neuen Thermostaten zu einem der chemischen oder spektrographischen Analyse hinsichtlich Einfachheit überlegenen Meßverfahren wird.
\end{abstract}

\section{Zweck und Nutzen des Kryostaten}

$\mathrm{N}$ achstehend wird ein vielseitig verwendbarer, zweistufiger Ganzmetall-Kryostat beschrieben, in dem flüssige Luft geeigneter Zusammensetzung un- ter vermindertem Druck eutektisch erstarrt, wodurch einfach, leicht und betriebssicher Temperaturen von $88^{\circ}$ bis hinab zu $50^{0}$ abs erreicht werden können.

Für Laboratorien, die über flüssigen $\mathrm{H}_{2}$ verfügen, bietet er die Möglichkeit, in dem sonst schwer zu- 